\title{
Altered Central-cardiovascular Network Pattern in Neuropathological Disease - Application of the Three Dimensional High Resolution Joint Symbolic Dynamics
}

\author{
Steffen Schulz ${ }^{1}$, Minia Ricoy ${ }^{2}$ Castro, Beatriz Giraldo ${ }^{3}$, Jens Haueisen ${ }^{4}$, Karl-Jürgen Bär ${ }^{5}$, Andreas \\ Voss $^{1}$ \\ ${ }^{1}$ Institute of Innovative Health Technologies, University of Applied Sciences, Jena, Germany \\ ${ }^{2}$ Department of Information and Communication Technologies, Universitat Pompeu Fabra, \\ Barcelona, Spain \\ ${ }^{3}$ ESAII Department, Barcelona East School of Engineering (EEBE), Technical University of \\ Catalonia (UPC), Barcelona, Spain \\ ${ }^{4}$ Institute of Biomedical Engineering and Informatics, University of Technology, Ilmenau, Germany \\ ${ }^{5}$ Department of Psychiatry and Psychotherapy, Pain and Autonomics-Integrative Research, \\ University Hospital, Jena, Germany
}

\begin{abstract}
The new interdisciplinary field of network physiology is getting more and more into the focus of interest in medicine. In this study, we introduce a new tool to analyse three dimensional couplings - the multivariate high resolution joint symbolic dynamics (mHRJSD) based on a redundancy reduction strategy and the analysis of dynamic processes by means of symbols. The mHRJSD is characterized by three symbols, a threshold for time series transformation, and 8 coupling pattern families.

In a first study mHRJSD was applied to 17 patients suffering from paranoid schizophrenia (SZ) and 17 healthy subjects (CO) to investigate short-term instantaneous central-cardiovascular network patterns. Therefore, from all participants, continuous heart rate (successive beat-tobeat intervals - BBI), non-invasive continuous systolic blood pressure (SYS) and power of the frontal 24-channel EEG $\left(P_{E E G}\right)$ were recorded for 15 minutes under resting conditions. We found highly significantly reduced complexity of the central-cardiovascular network in SZ and a reduced coupling of the central-cardiac network compared to CO. This study shows for the first time impaired complex central-cardiovascular network patterns in a neuropathological disease and might provide greater insight into the complex relationship between psychotic stages and autonomic activity.
\end{abstract}

\section{Introduction}

The new interdisciplinary field of network physiology is getting more and more into the focus of interest in medicine. It aims to define healthy and diseased states by analysing structural, dynamical and regulatory alterations in the interaction of physiological systems and subsystems. The central control of autonomic nervous system (ANS) and the complex interplay of its components can be described by a functional integrated mode - the centralautonomic-network (CAN). CAN represents the integrated function and interaction between, the central nervous system (CNS) and ANS (parasympathetic and sympathetic activity). It has been assumed that various autonomic function processes are generated by a network of interaction showing specificity for task and autonomic division. For healthy ones, Beissner et al. [1] suggested that asymmetric frontal EEG responses to emotional arousal in the form of positive and negative emotions may elicit different patterns of cardiovascular reactivity. In this study, we investigated for the first time the CAN under resting conditions in the neuropathological disease schizophrenia.

The multivariate coupling analysis of heart rate (HR), systolic blood pressure (SYS) and the power derived from the windowed electroencephalogram $\left(\mathrm{P}_{\mathrm{EEG}}\right)$ time series, respectively, might provide further information about the complex central-autonomic-network in neuropathological diseases than uni- and bivariate approaches can do. For the characterization of linear and nonlinear couplings several concepts are available [2] based on Granger causality, nonlinear prediction, entropies, symbolization, and phase synchronization that are able to detect direct and indirect couplings between time series. For the characterization of the beat-to-beat changes between HR, SYS and $\mathrm{P}_{\mathrm{EEG}}$ time series the new multivariate High Resolution Joint Symbolic Dynamics (mHRJSD) analysis approach was introduced based on the HRJSD [3].

The aim of this study was to quantify short-term nonlinear central-autonomic-network coupling pattern in 
acute schizophrenia applying mHRJSD analysis approach. We determines whether significant correlations existed between changes in EEG activity at the frontal lobe and changes in heart rate, as well as in systolic blood pressure.

\section{Materials and Methods}

\subsection{Subjects}

In this study, 17 healthy subjects (CO; 4 females, mean age $37.7 \pm 13.1$ years) and 17 patients suffering from paranoid schizophrenia (SZ; 2 females, $37.5 \pm 10.4$ years) were enrolled. Interviews and clinical investigations were performed for $\mathrm{CO}$ to exclude any potential psychiatric or other diseases. A structured clinical interview and a personality inventory (Freiburger Persönlichkeitsinventar) were also applied to the subjects to detect personality traits and any disorders which might influence autonomic function. All participants provided their written informed consent to a protocol approved by the local ethics committee of the Jena University Hospital. This study complies with the Declaration of Helsinki.

\subsection{Data recordings and pre-processing}

From all subjects, a 3-channel short-term ECG (500Hz), a non-invasive continuous blood pressure $(200 \mathrm{~Hz})$ and a 64-channel EEG $(500 \mathrm{~Hz})$ were recorded synchronously for 15 minutes. The EEG was acquired using 64 active $\mathrm{Ag} / \mathrm{AgCl}$ electrodes, and transmitted via a BrainAmp Amplifier (Brain Products, Germany; AFZ: ground, FCZ: reference). The electrodes were positioned according to the extended 10-20-system using an electrode cap. The impedance levels $(<25 \mathrm{~K} \Omega)$ for all electrodes were checked following the attachment of the electrode cap to each participant's scalp. The arterial blood pressure was recorded using the volume-clamp photoplethysmographical blood pressure device Portapres Model-2 (TNO Biomedical Instrumentation, Netherlands). All subjects' recordings were started after a supine resting period of 10 minutes. Subjects were asked to close their eyes, relax and breathe normally to avoid hyperventilation.

The following time series with respect to autonomous regulation were automatically extracted from the raw data records:

- Heart rate (lead I) consisting of successive beat-to-beat intervals (BBI, [ms]),

- Maximum successive systolic blood pressure amplitude values in relation to the previous R-peak (SYS, [mmHg]),

- Mean power $P_{\mathrm{EEG}}$ from the EEG (during each RRinterval, $\left.\left[\mu \mathrm{V}^{2}\right]\right)$.

EEG recordings (without any stimulation) were bandpass filtered $(0.05 \mathrm{~Hz}-60 \mathrm{~Hz}$, Butterworth filter, order $=3)$ in order to remove slow drifts resulting from slow body movements or sweating, and to prevent higher frequency content from additional noise. For EEG analyses, artefactfree time series from the frontal area (the frontal area (Fp1, Fp2, AF3, AF4, AF7, AF8, Fz, F1, F2, F3, F4, F5, F6, F7, F8, FC1, FC2, FC3, FC4, FC5, FC6, FT7, FT8, FT9, FT10) were used. All extracted time series (autonomous, central) were filtered by applying an adaptive variance estimation algorithm to remove and interpolate seldom occurring ventricular premature beats and artefacts (e.g., movement, electrode noise, and extraordinary peaks) to obtain normalto-normal beat time series (NN). To obtain synchronized time series, $\mathrm{BBI}, \mathrm{SYS}$, and $\mathrm{P}_{\mathrm{EEG}}$ were resampled using a linear interpolation method $(2 \mathrm{~Hz})$.

\subsection{Multivariate High Resolution Joint Symbolic Dynamics - mHRJSD}

HRJSD [3] was developed to analyse nonlinear cardiovascular couplings in acute schizophrenia treated with antipsychotics and based on the analysis of dynamic processes by means of symbols. In this study we applied an enhanced version - the mHRJSD - to quantify couplings of the central-autonomic-network. Therefore, three time series (BBI; SYS, $\mathrm{P}_{\mathrm{EEG}}$ ) were transformed into symbol sequences (Figure 1). $X$ as a trivariate sample vector, $x_{B B I}, x_{S Y S}$ and $x_{P E E G}$ were $n$ beat-to-beat values of $\mathrm{BBI}, \mathrm{SYS}$ and $\mathrm{P}_{\mathrm{EEG}}$, respectively.

$$
X=\left\{\left[x_{n}^{B B I}, x_{n}^{S Y S}, x_{n}^{P_{E E G}}\right]^{T}\right\}_{n=0,1, \ldots} \quad x \in R
$$

Afterwards, $X$ is transformed into a trivariate symbol vector $S$ defined as

$$
S=\left\{\left[s_{n}^{B B I}, s_{n}^{S Y S}, s_{n}^{P_{E E G}}\right]^{T}\right\}_{n=0,1, \ldots} s \in 0,1,2
$$

with the following definitions:

$$
\begin{aligned}
& S_{n}^{B B I}=\left\{\begin{array}{c}
0:\left(X_{n+1}^{B B I}-X_{n}^{B B I}\right)<-l^{B B I} \\
1:-l^{B B I} \leq\left(X_{n+1}^{B B I}-X_{n}^{B B I}\right) \leq l^{B B I} \\
2:\left(X_{n+1}^{B B I}-X_{n}^{B B I}\right)>l^{B B I}
\end{array}\right. \\
& S_{n}^{S Y S}=\left\{\begin{array}{c}
0:\left(X_{n+1}^{S Y S}-X_{n}^{S Y S}\right)<-l^{S Y S} \\
1:-l^{S Y S} \leq\left(X_{n+1}^{S Y S}-X_{n}^{S Y S}\right) \leq l^{S Y S} \\
2:\left(X_{n+1}^{S Y S}-X_{n}^{S Y S}\right)>l^{S Y S}
\end{array}\right.
\end{aligned}
$$

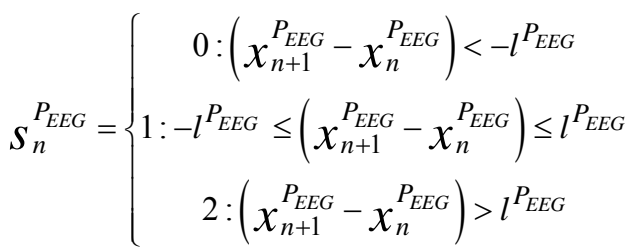


and the threshold levels $l_{B B I}=5 \mathrm{~ms}, l_{S Y S}=1 \mathrm{mmgHg}$ and $l_{\text {PEEG }}$ equal to $25 \%$ of the standard deviation of the $\mathrm{P}_{\mathrm{EEG}}$ time series as an adapted threshold to the individual physiological dynamic variability.



Figure 1. Principle of mHRJSD.

Transformation of the trivariate sample vector $X$ into the trivariate symbol vector $S$ ( 0 : decreasing values, 1 : equal values 2: increasing values); Word transformation and word pattern family distribution density matrix $W_{f}$ (8x8x8) with 8 pattern families E0, E1, E2, LU1, LD1, LA1, P, and V for SZ (average group values) with $p\left(w_{f}\right)>0.015$. $(\mathrm{BBI}=$ beat-to-beat intervals, $\mathrm{SYS}=$ systolic blood pressure amplitude over time, and $\mathrm{P}_{\mathrm{EEG}}=$ mean power of the EEG in relation to each RR-interval).

Symbol sequences with increasing values were coded as " 2 ", decreasing values were coded as ' 0 ' and unchanging (no variability) values were coded as ' 1 '. The symbol vector $S$ was subdivided into short words (bins) $w_{k}$ of length $k=3$. Thus, using three symbols led to 27 different word types for BBI $\left(w_{B B I}\right)$, SYS $\left(w_{S Y S}\right)$ and $\mathrm{P}_{\mathrm{EEG}}\left(w_{P E E G}\right)$. Afterwards, all single word types $w_{B B I, S Y S, P E E G}$ were grouped into 8 pattern families $w_{f}$ whereby the probabilities of these pattern families $p\left(w_{f}\right)$ were normalized to 1 . These 8 pattern families (E0, E1, E2, LU1, LD1, LA1, P, V) represent different aspects of centralautonomic modulation and were sorted into an $8 \times 8 \times 8$ pattern family density matrix $W_{f}$ resulting in 512 central- cardiovascular coupling patterns.

Pattern definition:

E0, E1 and E2: no variation within the word consisting of three symbols of type '0', '1' and'2', respectively.

LU1 and LD1: one variation within the word consisting of two different symbols with low increasing behaviour (LU1) and low decreasing behaviour (LD1).

LA1: one variation within the word consisting of two different alternating symbols of type ' 0 ' and ' 2 ' with an increasing-decreasing behaviour.

$\mathrm{P}$ and $\mathrm{V}$ : three variations within the word consisting of three different symbols with peak-like behaviour $(\mathrm{P})$ and with valley-like behaviour $(\mathrm{V})$.

In addition, from the matrix $W_{f}$ the Shannon entropy (mHRJSDshannon) and Renyi entropy (mHRJSDrenyi2, $\alpha=2$ ) as measures of the overall complexity of the CAN were computed.

\subsection{Statistics}

The nonparametric Mann-Whitney U-test was performed to evaluate differences between SZ and CO. Significances were considered for values of $* p<0.05$ and $* * p<0.01$. All results were presented as mean \pm std. Only coupling pattern with a probability of occurrence of $p(w f)>0.05$ were considered and had to fulfilled the Bonferroni-Holm adjustment $(* * * p<0.0000976)$.

\section{Results}

The mHRJSD method revealed three highly significant $(p<0.0000976)$ different central-cardiovascular network patterns between SZ and CO fulfilling the BonferroniHolm adjustment (Table 1). These three CAN pattern were dominated by two significant heart rate families pattern (BBI-E1, BBI-E2), one systolic blood pressure pattern (SYS-E1) and two mean power $\mathrm{P}_{\mathrm{EEG}}\left(\mathrm{P}_{\mathrm{EEG}}-\mathrm{LU} 1, \mathrm{P}_{\mathrm{EEG}^{-}}\right.$ LD1) from the EEG pattern and showed also univariate changes in SZ in comparison to CO (Table 1). In detail, we found in SZ an increased occurrence of the two CAN patterns that present an unchanged BBI and SYS with increased or decreased $\mathrm{P}_{\mathrm{EEG}}$ power (BBI-E1/SYS-E1/P $\mathrm{PEG}^{-}$

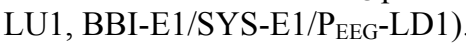

Moreover, the occurrence of the one CAN pattern that present an increased BBI, unchanged SYS and increased $P_{\text {EEG }}$ were decreased in SZ (BBI-E2/SYS-E1/PEEG-LU1). Thereby, the heart rate patterns were dominated by the families E2 and E1, the systolic blood pressure pattern by the family $\mathrm{E} 1$, and the mean power $\mathrm{P}_{\mathrm{EEG}}$ pattern were dominated by the families LU1 and LD1, respectively. In addition, we found a significantly increased occurrence of the one family representing unchanged BBI (BBI-E1), a significantly reduced occurrence of the one family representing increased BBI (BBI-E2) (heart rate $\downarrow$ ), and significantly increased occurrences of the two families 
representing increased or decreased $\mathrm{P}_{\mathrm{EEG}}\left(\mathrm{P}_{\mathrm{EEG}}-\mathrm{LU} 1, \mathrm{P}_{\mathrm{EEG}}-\right.$ LD1) in SZ compared to CO. Finally, we found significantly reduced complexity (mHRJSDshannon, mHRJSDrenyi2) of the CAN network in SZ compared to CO.

Table 1. Significant mHRJSD indices from centralcardiovascular coupling analysis between patients suffering from schizophrenia (SZ) and healthy controls (CO). $\left({ }^{*} p<0.05, * * p<0.01 ; * * * p<0.0000976\right)$

\begin{tabular}{|c|c|c|c|c|}
\hline & index & $p$ & CO & SZ \\
\hline & & & & \\
\hline 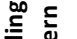 & BBI-E1/SYS-E1/P & $* * *$ & $0.018 \pm 0.027$ & $0.073 \pm 0.058$ \\
\hline 을 营 & BBI-E2/SYS-E1/P EEG $^{-L U 1}$ & $* * *$ & $0.051 \pm 0.027$ & $0.027 \pm 0.033$ \\
\hline & $\mathrm{BBI}-\mathrm{E} 1 / \mathrm{SYS}-\mathrm{E} 1 / \mathrm{P}_{\mathrm{EEG}}-\mathrm{LD} 1$ & $* * *$ & $0.016 \pm 0.024$ & $0.067 \pm 0.063$ \\
\hline & BBI-E2 & $* * *$ & $0.15 \pm 0.07$ & $0.06 \pm 0.07$ \\
\hline & BBI-E1 & $* * *$ & $0.08 \pm 0.10$ & $0.37 \pm 0.31$ \\
\hline 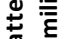 & SYS-E1 & n.s. & $0.74 \pm 0.18$ & $0.74 \pm 0.19$ \\
\hline & $P_{\mathrm{EEG}}-\mathrm{LU} 1$ & $* * *$ & $0.29 \pm 0.04$ & $0.30 \pm 0.04$ \\
\hline & $\mathrm{P}_{\mathrm{EEG}}-\mathrm{LD} 1$ & $* *$ & $0.29 \pm 0.03$ & $0.29 \pm 0.04$ \\
\hline 응 & mHRJSDshannon & $* * *$ & $5.6 \pm 0.6$ & $5.2 \pm 0.8$ \\
\hline 艺 & mHRJSDrenyi2 & $* * *$ & $4.8 \pm 0.6$ & $4.3 \pm 0.8$ \\
\hline
\end{tabular}

\section{Discussion}

We found highly significantly reduced complexity of the central-cardiovascular network in SZ and a reduced coupling of the central-cardiac network compared to CO. These diminished closed-loop interaction in SZ are probably caused by the known significant heart rate changes. It is presumed that lesions within the CNS may result in profound alterations in cardiac regulation and may even result in potentially fatal cardiac arrhythmias or sudden cardiac death (due to cardiovascular dysfunctions) [4]. The output of the CAN is directly linked to HRV. In addition, sensory information from end organs such as the heart and the immune system are feedback to the CAN, one important example of which is the baroreceptor reflex. As such, HRV is an indicator of central-peripheral neural feedback and CNS-ANS integration [5]. In this regard, it must be considered that the interactions between the CNS and ANS can be assumed to be a feedback-feedforward system which supports flexible and adaptive responses to environmental demands.

Limitations: We investigated the CAN under resting conditions whereas other studies are related to oddball paradigms with results on target and standard stimuli, working paradigm and simple auditory and visual-evoked potentials which make a direct comparison of these studies with this one difficult. Further on, SZ were treated with antipsychotics.

This study clearly shows an impaired brain-heart network that is expressed as a changed coupling between central activity and cardiovascular regulation in SZ patients. In addition, this study provides more detailed information about the interplay of neuronal and autonomic regulatory processes in SZ and most likely greater insight into the complex relationship between psychotic stages and autonomic activity.

\section{Acknowledgements}

This work was partly supported by grants from the Federal Ministry for Economic Affairs and Energy (BMWI) KF 2447308KJ4 and KF 2447309KJ4.

\section{References}

[1] Beissner F, Meissner K, Bar KJ and Napadow V. The autonomic brain: an activation likelihood estimation metaanalysis for central processing of autonomic function. $\mathrm{J}$ Neurosci 2013;33:10503-11.

[2] Schulz S, Adochiei FC, Edu IR, et al. Cardiovascular and cardiorespiratory coupling analyses: a review. Philos Trans A Math Phys Eng Sci 2013;371:20120191.

[3] Schulz S, Tupaika N, Berger S, Haueisen J, Bär KJ and Voss A. Cardiovascular coupling analysis with high-resolution joint symbolic dynamics in patients suffering from acute schizophrenia. Physiol Meas 2013;34:883-901.

[4] Foster PS and Harrison DW. The covariation of cortical electrical activity and cardiovascular responding. Int $\mathrm{J}$ Psychophysiol 2004;52:239-55.

[5] Thayer JF. What the Heart Says to the Brain (and vice versa) and Why We Should Listen. Psychological Topics 2007;16:241-50.

Address for correspondence.

Prof. Dr. Andreas Voss

Ernst-Abbe-Hochschule Jena, University of Applied Sciences Jena Institute of Innovative Health Technologies IGHT

Carl-Zeiss-Promenade 2, 07745 Jena, Germany

phone: 49-3641-205-625

E-Mail: andreas.voss@eah-jena.de 